\title{
La capacidad de las Organizaciones de la Sociedad Civil para mejorar la calidad de vida de los adultos mayores: un estudio cualitativo en zonas urbanas empobrecidas de México
}

\author{
The capacity of Civil Society Organizations to improve \\ the quality of life of the elderly: a qualitative study in \\ impoverished urban areas of Mexico
}

Tonatiuh T. González Vázquez1, Margarita Márquez Serrano², Sandra Treviño Siller ${ }^{3}$, Jesica Gómez-Jauregui ${ }^{4}$, Blanca Estela Pelcastre-Villafuerte ${ }^{5}$

\footnotetext{
${ }^{1}$ Licenciado en Ciencias Políticas. Doctorando en Ciencias Políticas, Centro de Investigación y Docencia en Humanidades del Estado de Morelos, México. Investigador en Ciencias Médicas, Instituto Nacional de Salud Pública, México.tgonzal@insp.mx

2Licenciada en Economía. Magíster en Medicina Social, Universidad Autónoma Metropolitana-Xochimilco, México. Investigadora en Ciencias Médicas, Instituto Nacional de Salud Pública, México.

mmarquez@correo.insp.mx

3 Licenciada en Antropología. Doctora en Antropología, Universidad Autónoma de México. Investigadora en Ciencias Médicas, Instituto Nacional de Salud Pública, México.strevino@insp.mx

${ }^{4}$ Licenciada en Ciencia Política. Doctora en Ciencias en Salud Pública, Instituto Nacional de Salud Pública, México. Consultora independiente. jesicagj@gmail.com

${ }^{5}$ Licenciada en Psicología. Doctora en Psicología Social, Universidad Autónoma de Barcelona. Investigadora en Ciencias Médicas, Instituto Nacional de Salud Pública, México.

bpelcast@correo.insp.mx
}

RESUMEN Recientemente en México se ha incrementado la proporción de adultos mayores (AM) y de Organizaciones de la Sociedad Civil (OSC) que ayudan a los pobres. Nuestro objetivo fue analizar la capacidad que tienen las OSC de implementar acciones para mejorar la calidad de vida de los AM pobres de zonas urbanas. En 2005 se realizaron 14 entrevistas al personal de diez OSC que trabajaban en colonias pobres de cuatro ciudades de México. La guía de entrevista y el análisis se basaron en la estructura interna y el contexto externo que afecta la capacidad de las OSC. Entre los principales logros se destacan una mayor participación y autogestión de la gente y el número de acciones realizadas para mejorar la calidad de vida de las personas. Los principales obstáculos identificados son una demanda poblacional que supera la capacidad de respuesta de las OSC, una población objetivo que generalmente no son los AM y un financiamiento insuficiente. La atención proporcionada por las OSC a los AM, aunque trascendental, es escasa, por tanto es necesario promover una cultura de prevención e interés en los AM y de apoyo a las OSC.

PALABRAS CLAVE Organizaciones No Gubernamentales; Anciano; Pobreza; Calidad de Vida; México.

\begin{abstract}
Recently, the proportion of elderly people (EP) has increased considerably in Mexico, as well as the number of Civil Society Organizations (CSOs) dedicated to helping poor people. Our objective was to analyze the capacity of the CSOs to implement actions in order to improve the quality of life of the poor EP in urban areas. In 2005, fourteen interviews were conducted with personnel of ten CSOs who worked in poor zones of four Mexican cities. The interview guide and the analysis were based on the internal structure and the external context that affect CSO's capacity. Within the main achievements, we identified an the increase in the participation and self-management of people and in the number of actions carried out in order to improve quality of people's life. The main obstacles identified were: the CSOs responsiveness, which is always lower than the population demands; their target population is not only EP; and insufficient financing. The attention provided by CSOs to EP is scarce, but essential. It is necessary to promote a culture of prevention and interest in the EP, and support to CSOs.
\end{abstract}

KEY WORDS Non-Governmental Organizations; Aged; Poverty; Quality of Life; Mexico. 


\section{INTRODUCCIÓN}

Dos fenómenos importantes se han venido desarrollando en el mundo a lo largo de los últimos 30 años: una transición demográfica que ha incrementado considerablemente la proporción de adultos mayores de 60 años y más (AM) y una mayor presencia de la sociedad civil (SC), que ha ganado espacios de expresión y de acción.

Aunque ambos fenómenos pueden continuar, y de hecho tienen un devenir independiente, debe ser de interés para la salud pública conocer hasta dónde ha llegado la acción de la SC en atención a las necesidades de ciertos grupos vulnerables de la sociedad, como los AM empobrecidos. Lo anterior, en virtud de que en este momento estamos viviendo un acelerado proceso de envejecimiento de la población, de manera que el sistema público de salud en México se ha visto rebasado por el incremento de las necesidades de este grupo poblacional y las acciones y políticas dirigidas a este sector no pueden, de ningún modo, postergarse. En este interés se basa el presente estudio.

\section{EVOLUCIÓN DE LA SOCIEDAD CIVIL ORGANIZADA}

Durante las últimas décadas del siglo pasado las distintas versiones del Estado que regulaban la economía para compensar las desventajas sociales de ciertos grupos -benefactor en Europa occidental y Norteamérica, populista en América Latina y socialista en la URSS y Europa del Este- entraron en crisis (1), entre otras cosas debido a la pérdida de eficacia de las tradicionales fórmulas de gestión económica y social de orden corporativo y clientelar y el cuestionamiento público del universo de los políticos por motivos de corrupción y nepotismo (2).

Intelectuales e integrantes de la SC de izquierda y derecha coincidían en que era necesario transformar la institucionalidad pública: mientras que unos proponían renovarla otros sugerían que se limitara. Tanto la respuesta neoliberal como la estatal fueron insuficientes y esta coyuntura facilitó el crecimiento exponencial de la SC y de las Organizaciones de la Sociedad
Civil (OSC). Estas últimas se encargaron de la producción de servicios públicos, la cual fue apoyada por instituciones multilaterales como el Banco Interamericano de Desarrollo y el Banco Mundial $(3,4)$.

Actualmente la SC es vista como el lugar de la legitimidad democrática y de los derechos en donde los individuos hablan, se asocian y razonan juntos sobre asuntos de interés público, con el fin de influir indirectamente en la toma de decisiones (5). La SC tiene distintas formas de expresarse y organizarse, desde movimientos sociales, espontáneos y esporádicos, hasta organizaciones más institucionalizadas y permanentes como pueden ser las OSC, también conocidas como Organizaciones no Gubernamentales. Salamon las define como organizaciones privadas de interés público que persiguen objetivos públicos fuera del aparato formal del Estado, que son normalmente voluntarias y que no tienen como fin principal el lucro (6).

Las principales áreas de concentración de las OSC son educación, salud, servicios sociales, y actividades recreativas y culturales (7). Torres retoma a Korten quien clasifica a las OSC de acuerdo a sus estrategias de acción, identificando tres generaciones:

- Primera: remedian las necesidades más inmediatas de la población mediante acciones directas como distribución de comida y brigadas de salud; son las Ilamadas asistencialistas.

- Segunda: generan la autoestima y capacidad para que los miembros de la comunidad obtengan beneficios aun después de que se les dio la ayuda. Apoyan proyectos de desarrollo comunitario.

- Tercera: intentan crear una base nacional o regional para lograr la sustentabilidad del cambio en las comunidades. Interactúan con una mayor cantidad de organizaciones públicas y privadas que controlan los recursos y las políticas de desarrollo, lo cual ha hecho necesaria la vinculación con el gobierno (6).

Dentro de las OSC es determinante la disposición de financiamiento para alcanzar sus objetivos, el cual obtienen principalmente de donaciones, cooperación internacional y, 
últimamente, del gobierno y a través de la prestación de servicios. Si bien una de las características del sector son los ideales y la motivación del personal, estos no son suficientes, ya que cada vez es más necesaria una profesionalización del mismo (8).

Tan solo en 36 países estudiados, durante el período 1995-2000, este sector movilizó un total de 1,3 trillones de dólares, ocupando a 45,5 millones de trabajadores de tiempo completo y a 132 millones de voluntarios. México ocupó el último lugar en cuanto a población económicamente activa empleada en el sector, con un 0,4\% (7).

En el país, el crecimiento exponencial de las OSC se inicia en la década del '70 y continúa en los '80 y '90. A pesar de ser considerado como uno de los sectores más reducidos de Latinoamérica, en 1995 las OSC nacionales tuvieron un gasto operativo de 1.300 millones de dólares (8.800 millones de pesos mexicanos), con una fuerza laboral de 93.809 empleados asalariados y 47.000 voluntarios (9). Este crecimiento se caracterizó por un bajo nivel de supervivencia de las mismas, el cual está relacionado a la falta de recursos financieros, un bajo nivel de profesionalización y divergencias entre sus miembros (10).

Esta situación de constante creación y desaparición de OSC dificulta que se tengan datos precisos y actualizados sobre su número, distribución, temática e impacto. Uno de los esfuerzos más sistematizados al respecto fue el Censo Nacional de Organizaciones Civiles, de acuerdo al cual en el año 2000 existían en el país 9.013. En cuanto a la distribución de las OSC en México, el Distrito Federal ocupaba el primer lugar con el 16,55\% (1.492), seguido del estado de México con 5,67\% (511) y Jalisco en el tercero con $5,45 \%$ (491). Además, los otros tres estados en los que realizamos el presente estudio fueron: Sinaloa, que ocupa el lugar diecisiete con $2,87 \%$ (259), Morelos en el lugar dieciocho con $2,77 \%$ (250) y Guerrero con el 1,46\% (132), en el lugar veinticinco (10).

\section{TRANSICIÓN DEMOGRÁFICA: CADA VEZ MÁS ADULTOS MAYORES}

Actualmente el mundo está viviendo las últimas etapas de una larga transición demográfica.
Se calcula que para el año 2050, la proporción mundial de AM alcanzará más del doble de la del año 2000, pasando de aproximadamente el $7 \%$ (418 millones) al 16\% (1.440 millones) de la supuesta futura población mundial de 9.000 millones de personas (11). México ocupa el séptimo lugar entre los países con envejecimiento acelerado en Latinoamérica. El Consejo Nacional de Población (12) estimó que en 2006 había en el país 9,6 millones de personas de 60 años y más, que representaban el 9,3\% de la población total. De acuerdo a sus proyecciones, en 2050 se estima que habrá 36 millones de AM, lo que representará el $28 \%$ de la población total mexicana (13). Para el año 2001 aproximadamente el $44 \%$ de los AM vivía en comunidades urbanas (14), que es donde se concentran la mayoría de las acciones de las OSC.

El contexto social, económico y cultural en el que está ocurriendo el envejecimiento poblacional en Latinoamérica se caracteriza por una alta incidencia de pobreza, una persistente y aguda inequidad social, la baja cobertura de la seguridad social y una probable tendencia hacia el deterioro de las estructuras familiares de apoyo al anciano (15), todo lo cual va en detrimento de su calidad de vida. En México, esta situación de desprotección social es similar: datos de CONAPO (12) indican que en el año 2005 el 44\% de los AM no contaba con derechohabiencia a servicios de salud; en un estudio previo (16) realizado en las mismas ciudades que las del estudio que aquí se presenta, se encontró que este porcentaje es mayor $(54,9 \%$ ) y que tan solo el $6,6 \%$ contaba con el Seguro Popular (a). La ayuda institucional fue aun menor, pues solamente el 13,4\% recibe beneficios de algún programa gubernamental y únicamente el 11,6\% cuenta con una jubilación o pensión. La cuarta parte de ellos recibía recursos económicos por parte de sus familiares y el $55 \%$ de los AM no reciben ni ayuda económica familiar ni jubilación o pensión.

Estos indicadores numéricos dan cuenta de la magnitud de la desprotección de esta población, y están directamente vinculados con la calidad de vida de los AM. Para la Organización Mundial de la Salud (OMS), la calidad de vida es la autopercepción que tiene un individuo de su situación en la vida dentro del contexto cultural y de los valores en los que vive, y en relación con 
sus objetivos, expectativas, patrones, preocupaciones e intereses (17). Dentro de los aspectos objetivos de la calidad de vida están los determinantes sociales, demográficos, políticos y económicos; mientras que los aspectos subjetivos se refieren a la percepción que la persona tiene de su salud y de su bienestar psicológico (18).

A falta de recursos profesionales, técnicos y económicos, el Estado en México no está dando una solución completa a los problemas que genera el incremento de los AM y difícilmente lo podrá hacer en el futuro, por eso el apoyo familiar y de la SC es fundamental para contribuir a mejorar su calidad de vida. Es importante tener claro que no se propone que las OSC sustituyan al Estado y sus políticas sociales, ya que éste es fundamental para la promoción y protección de los derechos sociales, teniendo como responsabilidad el bienestar general de la población $(3,4,19)$.

Lo que Cunill propone es que las OSC jueguen un rol más activo y sean corresponsables, junto con el Estado, en la producción de servicios sociales. Entre los atributos de las OSC se encuentran una mayor flexibilidad, la disposición de experiencia especializada y la habilidad para llegar a públicos de difícil acceso y marginados como los migrantes y AM. Pero el Estado debe tener la capacidad de distinguir bajo qué situaciones es posible y conveniente la participación de las OSC en los servicios sociales, y cuándo no lo es, ya sea porque no existe un tejido social fuerte o porque el desempeño es deficiente, ya que en muchos países, las OSC son aún nuevas y/o débiles a tal punto que apelar a su colaboración puede provocar más vulnerabilidad a la política social (4).

En el año 2005, de las 10.625 OSC registradas en el directorio del Centro Mexicano para la Filantropía (CEMEFI), 273 otorgaban servicios enfocados a los AM (20), número que parece bajo si consideramos el creciente incremento de esta población. Bajo este panorama, sería importante estudiar estas OSC para determinar si podrán contribuir sustancialmente a hacer frente a los retos que plantea la transición demográfica para el futuro inmediato; no obstante, un paso previo sería el conocimiento de sus acciones y las facilidades y obstáculos a los que se enfrentan en esta tarea. El objetivo de este estudio fue analizar la capacidad que tienen las OSC de Ilevar a cabo acciones para mejorar la calidad de vida de la población adulta mayor en zonas urbanas empobrecidas.

\section{MATERIAL Y MÉTODOS}

Como parte del proyecto Salud y calidad de vida en adultos mayores viviendo en la pobreza urbana, se diseñó un estudio cualitativo realizado entre octubre y diciembre del 2005 con OSC que trabajaban con AM en colonias pobres de las ciudades de Guadalajara, estado de Jalisco; Culiacán, estado de Sinaloa; Cuernavaca, estado de Morelos y Chilpancingo, estado de Guerrero. Del año 2001 al 2003, con excepción de Sinaloa, se realizaron investigaciones con AM pobres de zonas rurales (21). Entre 2003 y 2005 se desarroIló otro estudio, con una aproximación cuantitativa, que se enfocaba en los AM de zonas urbanas de estas cuatro ciudades (22). De esta manera, las ciudades fueron seleccionadas buscando la diversidad (geográfica, cultural, económica y política) existente en el país y considerando el criterio de continuidad con el trabajo de investigación previamente realizado, más que por el número de OSC enfocadas en AM urbanos. Esta información fue indagada posteriormente, a través de una búsqueda temática en el directorio del CEMEFI y en el Sistema de Información sobre OSC del Instituto Nacional de Desarrollo Social. Esta información se complementó de manera telefónica y con visitas a las colonias marginadas para buscar OSC que no aparecieran en los directorios. Para tener una muestra plural, se entrevistó solo un asilo, pese a que son las OSC que más trabajan con AM. Debido a la escasez de OSC enfocadas en AM urbanos, también se identificaron organizaciones que trabajaran con población abierta de las colonias pobres urbanas, encontrando una gran variación en el porcentaje de AM a los cuales benefician.

Al final se trabajó con diez OSC en las que siempre se entrevistó a un directivo y, en los casos en los que esto fue posible, además se entrevistó a una persona operativa. En total se realizaron 14 entrevistas semiestructuradas; las entrevistas se distribuyeron de la siguiente manera: tres en Cuernavaca, cinco en Guadalajara, 
cinco en Culiacán y una en Chilpancingo. En esta última ciudad se entrevistó únicamente una organización debido a que fue la única OSC identificada que trabajaba con población urbana pobre. Todos los participantes firmaron una carta de consentimiento después de haber sido informados sobre los objetivos del estudio y asegurárseles el anonimato. Las entrevistas fueron grabadas y transcriptas para su análisis a través de matrices de datos. La guía de entrevista y el análisis se basaron en la propuesta de Gómez-Jauregui (23), quien considerando diferentes abordajes conceptuales, diseñó un modelo de análisis de las OSC que explora la estructura interna (misión, objetivos y acciones; recursos humanos; toma de decisiones, cultura organizacional y estructura financiera) y el contexto externo (económico, político, social, institucional-legal y las redes) que afectan su capacidad. Estos elementos constituyeron las categorías analíticas del estudio.

El protocolo del estudio fue aprobado por las Comisiones de Investigación y Ética del Instituto Nacional de Salud Pública de México. Para evitar que se pudiera reconocer a qué OSC pertenecía el personal cuyos testimonios se incluyeron, se omitió cualquier dato que pudiera identificarlos.

\section{RESULTADOS}

Todos los directivos señalaron que la población objetivo de su OSC son las personas pobres a las cuales otorgan ayuda para mejorar su calidad de vida. La mayoría de las OSC tenía como estrategia apoyar a gente que no fuera beneficiaria de algún programa de gobierno. Únicamente tres de las OSC (Banco de Alimentos de Culiacán, Voluntariado Estamos Contigo y Promotores para el Desarrollo de la Empresa Social) no realizaban acciones permanentes en temas de salud. Todas las OSC trabajaban con población urbana; a pesar de ello, dos trabajaban mayoritariamente en zonas rurales (Banco de Alimentos de Culiacán y Promotores para el Desarrollo de la Empresa Social).

Con base en las estrategias utilizadas por las OSC señaladas por Torres (6), tenemos de primera generación al Banco de Alimentos de
Culiacán y al Club de Amigos de la Tercera Edad, que son asistencialistas, a través del otorgamiento de despensas y asilo a AM. Seis de las OSC entrevistadas se acercan más a la categoría de segunda generación pues combinan el asistencialismo (médico y alimentario) con capacitación (técnica y espiritual), educación para la salud, investigación, rehabilitación y tanatología. Solo dos de las OSC podrían aproximarse a la categoría de tercera generación; ambas tienen una base regional sólida: Promotores para el Desarrollo de la Empresa Social busca la sustentabilidad del cambio a partir del otorgamiento de microcréditos para negocios, mientras que el Voluntariado Estamos Contigo estaba en proceso de hacerlo, pero ambas han realizado un escaso acercamiento al gobierno (Cuadro 1 y 2).

El promedio del personal laboral en las OSC fue de 18 personas; las mujeres tenían una participación mayoritaria en ellas y la media de edad fue de 35 años. Las acciones de las diez OSC beneficiaban a los AM, pero solo tres trabajaban principalmente con ellos y únicamente el Club de Amigos de la Tercera Edad se dedicaba exclusivamente a este grupo poblacional (Cuadro 1).

\section{LOGROS Y LIMITACIONES EN LA CAPACIDAD DE LA ORGANIZACIÓN}

A continuación se analizan con detalle algunos elementos que limitan o favorecen la capacidad de las OSC, entendida como la habilidad para alcanzar sus objetivos o cumplir con su misión de manera efectiva (24), los que repercuten en su desempeño.

La misión de las OSC busca básicamente mejorar la calidad de vida de la población pobre a través de las siguientes acciones: proveer alimentos, útiles escolares, asilo, alfabetización, capacitación técnica y en valores humanos, además de microcréditos. Uno de los temas más constantes es la salud física y mental a través de consultas tanatológicas, médicas y psicológicas, cirugías, medicamentos, educación, prevención y rehabilitación (Cuadro 1-4).

En cuanto al número de personas beneficiadas anualmente, se observaron variaciones de 29 a 32.226 personas. En seis de las diez OSC las empresas privadas daban un financiamiento 


\begin{tabular}{|c|c|c|c|c|c|c|c|c|}
\hline \multirow[t]{2}{*}{ NOMBRE } & \multirow[t]{2}{*}{ MISIÓN } & \multirow[t]{2}{*}{ ACCIONES } & \multicolumn{2}{|c|}{ PERSONAL } & \multicolumn{2}{|c|}{$\begin{array}{l}\text { PERSONAS BENEFICIADAS } \\
\text { (anualmente) }\end{array}$} & \multirow[t]{2}{*}{$\begin{array}{l}\text { INSTANCIAS DE } \\
\text { FINANCIAMIENTO }\end{array}$} & \multirow[t]{2}{*}{$\begin{array}{l}\text { GASTO ANUAL } \\
\text { (\$ mexicanos) }\end{array}$} \\
\hline & & & Total & $\begin{array}{l}\text { Edad } \\
\text { (prom. años) }\end{array}$ & Total & $\begin{array}{l}\text { Adultos mayores } \\
(\%)\end{array}$ & & \\
\hline $\begin{array}{l}\text { Voluntariado } \\
\text { Estamos } \\
\text { Contigo }\end{array}$ & $\begin{array}{l}\text { Dar cursos de } \\
\text { superación huma- } \\
\text { na para contribuir } \\
\text { a un México mejor. }\end{array}$ & $\begin{array}{l}\text { Capacitación } \\
\text { técnica y en valores } \\
\text { humanos y despensas } \\
\text { alimenticias. }\end{array}$ & 212 & 30 & 11.000 & 5,0 & $\begin{array}{l}\text { Un solo donador de la } \\
\text { iniciativa privada. }\end{array}$ & $\$ 3.600 .000$ \\
\hline $\begin{array}{l}\text { Fundación } \\
\text { Pro-vista }\end{array}$ & $\begin{array}{l}\text { Dar atención } \\
\text { médica en enfer- } \\
\text { medades de los } \\
\text { ojos a todo tipo de } \\
\text { personas, en } \\
\text { especial de } \\
\text { escasos recursos. }\end{array}$ & $\begin{array}{l}\text { Servicios médicos } \\
\text { oftalmológicos: consulta, } \\
\text { cirugía, óptica, farma- } \\
\text { cia, campañas de } \\
\text { información sobre } \\
\text { enfermedades de los } \\
\text { ojos y detección de } \\
\text { glaucoma. }\end{array}$ & 36 & 35 & 32.226 & 80,0 & Prestación de servicios. & $\$ 10.000 .000$ \\
\hline $\begin{array}{l}\text { Club de } \\
\text { Amigos de } \\
\text { la Tercera } \\
\text { Edad }\end{array}$ & $\begin{array}{l}\text { Velar por el } \\
\text { adulto mayor, } \\
\text { sobre todo } \\
\text { indigente. }\end{array}$ & $\begin{array}{l}\text { Guardería diurna para } \\
\text { adutos mayores de } \\
\text { clase media y alta, y } \\
\text { asilo para adultos } \\
\text { mayores indigentes. } \\
\text { Atención médica y de } \\
\text { enfermería. }\end{array}$ & 12 & 30 & 29 & 100,0 & $\begin{array}{l}\text { Aportaciones de } \\
\text { los miembros. } \\
\text { Cuota de recuperación. } \\
\text { Donaciones. } \\
\text { Iniciativa privada. } \\
\text { Ciudadanos. } \\
\text { OSC estatal. } \\
\text { Instituto Jalisciense } \\
\text { de Asistencia Social. } \\
\text { Gubernamental. } \\
\text { Fundación nacional. }\end{array}$ & $\$ 240.000$ \\
\hline
\end{tabular}

Fuente: Elaboración propia.

considerable que sostenía parte de sus acciones, cuyo gasto anual suele superar el millón de pesos, hasta alcanzar los doce millones. En conjunto las diez OSC tenían un gasto anual de \$39.790.000 pesos, beneficiando a un total de 82.963 personas (Cuadro 1-4). Es necesario aclarar que el impacto temporal es diferencial, existiendo OSC que generalmente atienden a la gente en una sola ocasión, como las dos clínicas de salud visual, mientras que otras, como el Banco de Alimentos de Culiacán y el Voluntariado Estamos Contigo, otorgan ayuda por varios meses. Por último tenemos al Club de Amigos de la Tercera Edad, que si bien solo beneficia a 29 AM, la ayuda es permanente hasta que las personas mueren.

La capacidad de las diez OSC ha sido, en general, positiva; empero, han enfrentado situaciones que limitan su desempeño. Dentro de los logros, las OSC mencionaron: el incremento en la participación y autogestión de la gente, el empoderamiento de la población y el hecho de crear la idea de que se pueden cambiar y mejorar las condiciones sociales; pero aclararon que estos cambios se han logrado después de un trabajo arduo.

Con respecto a las limitaciones, dos OSC se vieron en la necesidad de elegir entre ayudar a las poblaciones urbanas o rurales, ya que no pueden trabajar con ambas, optando por las rurales, al percibirlas con mayores carencias o al existir impedimentos de las financiadoras para apoyar el trabajo con comunidades urbanas. Estas limitaciones de cobertura hacen que comúnmente se restrinja la difusión de sus servicios ya que su capacidad de respuesta se vería superada por la demanda de la población:

"Prácticamente aquí la difusión es de voz a voz entre la misma gente y con póster en el lapso de la capacitación, porque si nos vamos a radio, a medios de comunicación, no nos daríamos abasto, hace como dos años lanzamos un póster de los servicios que prestábamos y aquí teníamos Ileno." 
Algunas OSC reconocieron que tienen un impacto limitado porque no pueden cambiar algunos problemas estructurales de la sociedad como las adicciones, la pobreza y el acostumbramiento de la gente al asistencialismo:

"La pobreza y el hambre no se van a disminuir nada más porque estemos trabajando, la pobreza y el hambre crecen casi de manera demográfica. [...] Lo que hemos observado en estos 18 años es que las personas más se acostumbran a recibir que a dar. Vicios, adicciones y demás, esas siguen igual, no mejoran, por más que a través de los cursos se les trate de interesar en mejorar la situación [...] sí, algunos van saliendo pero es el número menor..."

A continuación se analizarán algunos de los elementos dentro de la organización y en su entorno, que por un lado han ampliado y por otro limitado la capacidad de las OSC para dar atención a los AM.

\section{ESTRUCTURA INTERNA}

Bajo esta categoría analizamos la estructura organizacional que tiene tres funciones básicas: 1) generar productos y alcanzar los objetivos de la organización; 2) regular la influencia de variaciones individuales, asegurando que los individuos actúen conforme a los requerimientos de la organización; y 3) analizar las características del contexto en el que se toman las decisiones y en el que se llevan a cabo las actividades de la organización (25). Como parte de los problemas dentro de la estructura interna se destacan la dificultad que tienen algunas OSC para incorporar personal de tiempo completo así como la falta de profesionalización de la organización.

Aunque con un mayor énfasis en las OSC de Cuernavaca y Chilpancingo, el principal problema interno reconocido por la mayoría de las organizaciones fue el financiamiento, destacando su escasez, la dificultad para acceder a los recursos, el cansancio de las financiadoras con las OSC y la falta de apoyo del gobierno para acceder a mayores recursos:

"Sí, cuando la fundación de Holanda dejó de apoyarnos, incluso tuvimos que recurrir a la buena voluntad de los compañeros y como seis meses no se les pagó nada hasta que se volvió a restablecer el apoyo financiero."

También se mencionó que algunas financiadoras condicionaban los recursos para ser aplicados en poblaciones específicas (niños y hombres). En una de las OSC, pese a que en un inicio se trabajaba únicamente con $\mathrm{AM}$, en la actualidad solo el $30 \%$ de sus actividades son dirigidas a este grupo, debido a que una de las financiadoras le condicionó los recursos para que trabaje también con niños.

Mención aparte merecen las dos clínicas de salud visual (Guadalajara y Cuernavaca), las cuales desarrollaron un sistema de financiamiento que las hace mayoritariamente autosuficientes,

Cuadro 2. CARACTERÍSTICAS DE LAS ORGANIZACIONES DE LA SOCIEDAD CIVIL QUE TRABAJAN CON POBLACIÓN URBANA EMPOBRECIDA. CHILPANCINGO, MÉXICO, 2004-2005.

\begin{tabular}{|c|c|c|c|c|c|c|c|c|}
\hline \multirow[t]{2}{*}{ NOMBRE } & \multirow[t]{2}{*}{ MISIÓN } & \multirow[t]{2}{*}{ ACCIONES } & \multicolumn{2}{|c|}{ PERSONAL } & \multicolumn{2}{|c|}{$\begin{array}{l}\text { PERSONAS BENEFICIADAS } \\
\text { (anualmente) }\end{array}$} & \multirow[t]{2}{*}{$\begin{array}{l}\text { INSTANCIAS DE } \\
\text { FINANCIAMIENTO }\end{array}$} & \multirow[t]{2}{*}{$\begin{array}{l}\text { GASTO ANUAL } \\
\text { (\$ mexicanos) }\end{array}$} \\
\hline & & & Total & $\begin{array}{l}\text { Edad } \\
\text { (prom. años) }\end{array}$ & Total & $\begin{array}{l}\text { Adultos mayores } \\
(\%)\end{array}$ & & \\
\hline $\begin{array}{l}\text { Promotores } \\
\text { para el } \\
\text { Desarrollo de } \\
\text { la Empresa } \\
\text { Social }\end{array}$ & No definida. & $\begin{array}{l}\text { Capacitación y } \\
\text { microcréditos para } \\
\text { empresas productivas, } \\
\text { en mujeres rurales y en } \\
\text { menor proporción } \\
\text { urbanas. }\end{array}$ & 5 & - & 14.000 & 35,0 & $\begin{array}{l}\text { Gubernamental. } \\
\text { Fundación nacional. }\end{array}$ & $\$ 2.500 .000$ \\
\hline
\end{tabular}

Fuente: Elaboración propia. 
CUadro 3. CARACTERÍSTICAS DE LAS ORGANIZACIONES DE LA SOCIEDAD CIVIL QUE TRABAJAN CON POBLACIÓN URBANA EMPOBRECIDA. CUERNAVACA, MÉXICO, 2004-2005.

\begin{tabular}{|c|c|c|c|c|c|c|c|c|}
\hline \multirow[t]{2}{*}{ NOMBRE } & \multirow[t]{2}{*}{ MISIÓN } & \multirow[t]{2}{*}{ ACCIONES } & \multicolumn{2}{|c|}{ PERSONAL } & \multicolumn{2}{|c|}{$\begin{array}{l}\text { PERSONAS BENEFICIADAS } \\
\text { (anualmente) }\end{array}$} & \multirow[t]{2}{*}{$\begin{array}{l}\text { INSTANCIAS DE } \\
\text { FINANCIAMIENTO }\end{array}$} & \multirow[t]{2}{*}{$\begin{array}{l}\text { GASTO ANUAL } \\
\text { (\$ mexicanos) }\end{array}$} \\
\hline & & & Total & $\begin{array}{l}\text { Edad } \\
\text { (prom. años) }\end{array}$ & Total & $\begin{array}{l}\text { Adultos mayores } \\
(\%)\end{array}$ & & \\
\hline $\begin{array}{l}\text { Asociación } \\
\text { Centro de } \\
\text { Rehabilitación } \\
\text { para Ciegos }\end{array}$ & $\begin{array}{l}\text { Promover la } \\
\text { cultura de la } \\
\text { prevención y } \\
\text { atención en } \\
\text { salud ocular en } \\
\text { la población } \\
\text { vulnerable. }\end{array}$ & $\begin{array}{l}\text { Servicios médicos } \\
\text { oftalmológicos: } \\
\text { consulta, cirugía, } \\
\text { óptica, farmacia, } \\
\text { campañas de } \\
\text { prevención. } \\
\text { Rehabilitación para } \\
\text { ciegos y débiles } \\
\text { visuales. }\end{array}$ & 50 & 35 & 5.722 & 26,0 & $\begin{array}{l}\text { Prestación de servicios. } \\
\text { Gubernamental. } \\
\text { OSC nacional. }\end{array}$ & $\$ 12.000 .000$ \\
\hline $\begin{array}{l}\text { Ancianos y } \\
\text { sus Amigos }\end{array}$ & $\begin{array}{l}\text { Apoyo asistencial } \\
\text { a adultos mayores } \\
\text { de escasos } \\
\text { recursos. }\end{array}$ & $\begin{array}{l}\text { Proveen despensas } \\
\text { alimenticias, útiles } \\
\text { escolares, consultas } \\
\text { médicas y tanatológicas } \\
\text { en los domicilios a } \\
\text { adultos mayores y niños. }\end{array}$ & 3 & 54 & 400 & 30,0 & $\begin{array}{l}\text { Fundación internacional. } \\
\text { Donador privado. } \\
\text { Ciudadanos. }\end{array}$ & $\$ 420.000$ \\
\hline $\begin{array}{l}\text { Centro } \\
\text { Comunitario } \\
\text { Infantil } \\
\text { Encuentro }\end{array}$ & $\begin{array}{l}\text { Fomentar la } \\
\text { concientización y } \\
\text { solidaridad entre } \\
\text { las personas para } \\
\text { ayudar a los más } \\
\text { necesitados. }\end{array}$ & $\begin{array}{l}\text { Desayunos, educación } \\
\text { y alfabetización para } \\
\text { niños. Cursos de inte- } \\
\text { gración familiar, banco } \\
\text { de medicamentos, } \\
\text { consulta médica y } \\
\text { psicológica y cursos } \\
\text { de manualidades para } \\
\text { adultos mayores. }\end{array}$ & 35 & 30 & 250 & 5,0 & $\begin{array}{l}\text { Cuota de recuperación. } \\
\text { Ciudadanos mexicanos } \\
\text { y extranjeros. } \\
\text { Gubernamental. } \\
\text { Escuelas de otro país. }\end{array}$ & $\$ 250.000$ \\
\hline
\end{tabular}

Fuente: Elaboración propia.

basado en cobrar a las personas con mayores ingresos económicos una cuota competitiva con las clínicas privadas por medio de la cual se pagan muchos de los gastos operativos y se subsidia a las personas de bajos ingresos, a quienes se le cobra solo una cuota de recuperación e incluso se les llega a dar el servicio de forma gratuita.

\section{CONTEXTO EXTERNO}

El entorno político en el que las OSC operan y la estructura de recursos a la que pueden acceder tienen consecuencias importantes para las estrategias internas. Un entendimiento adecuado de su funcionamiento requiere prestar atención a la relación entre la estructura organizacional interna y las características del entorno (26).

Hay poco interés de todos los sectores en dar atención a los AM, el gobierno les dedica pocos recursos y el interés del sector lucrativo se dirige a aquellos con capacidad de pago. Desde el punto de vista de los informantes, son pocas las OSC dedicadas exclusivamente a ayudar a los AM, pese a que es común que existan organizaciones enfocadas a otros grupos vulnerables. Se señaló que esto se debe, entre otras cosas, a una cultura de falta de prevención e interés en los AM:

"Yo creo que nos falta mucho de manera cultural como de prever qué va a pasar con toda la población que vamos a llegar a viejos. Entonces no hay una preocupación todavía por atender esta parte, [las OSC] se enfocan mucho a los niños, se enfocan mucho a mujeres. No ha habido la preocupación todavía por ver qué se les puede ofrecer [a los adultos mayores], lo más a lo que llegamos a nivel nacional es abrir asilos para que ahí se recluyan y ahí darles de comer como si eso fuera suficiente." 
En todas las ciudades, la coordinación con alguna instancia de gobierno se dio principalmente con el Sistema Nacional para el Desarrollo Integral de la Familia (DIF), y si bien se les reconocía esta colaboración, también se señaló que la ayuda se daba más de las OSC hacia el DIF que a la inversa. Esta especie de "subsidio" de las OSC se otorga también a otras instancias públicas y privadas:

[Los distintos gobiernos] "no nos toman en cuenta para nada. La clínica le ahorra a la población $\$ 1.200 .000$ pesos mensuales aproximadamente, si nuestros servicios estuvieran a los precios de los médicos privados. Estamos haciendo el trabajo que no hace el gobierno, dando servicios de calidad, de especialidad a bajo costo, cosa que no hacen los hospitales generales y todas esas personas vienen para acá, inclusive ellos nos mandan todas sus urgencias, todos los problemas que no pueden resolver, recibimos las urgencias del DIF, del Hospital del Niño, de todas las comunidades del estado."
En términos generales, las OSC de Guadalajara y Culiacán percibieron un mayor apoyo gubernamental. Esta percepción se ve reflejada en el reconocimiento que hacen estas OSC a la Junta de Asistencia Privada del estado de Sinaloa y al Instituto Jalisciense de Asistencia Social en su labor de fomentar las actividades del sector. Por su parte, las OSC de Cuernavaca y Chilpancingo no solo manifestaron falta de apoyo gubernamental a sus programas, con un apoyo financiero escaso, discrecional y con un exceso de trámites, sino también de las redes de OSC, partidos políticos e instancias privadas, las cuales les dificultan su funcionamiento:

"De alguna manera sí [nos afecta la situación política], porque al parecer aquí en el estado hay votaciones cada año, entonces hay una perversidad; hemos estado en reuniones con la gente $y$ creen que nosotros estamos haciendo un proselitismo político cuando no es así. En cuanto a los microcréditos, los partidos políticos luego le dicen a la gente: 'no pagues, ese dinero no se paga, es del gobierno', cuando no es así. [...] Los

Cuadro 4. CARACTERÍSTICAS DE LAS ORGANIZACIONES DE LA SOCIEDAD CIVIL QUE TRABAJAN CON POBLACIÓN URBANA EMPOBRECIDA. CULIACÁN, MÉXICO, 2004-2005.

\begin{tabular}{|c|c|c|c|c|c|c|c|c|}
\hline \multirow[t]{2}{*}{ NOMBRE } & \multirow[t]{2}{*}{ MISIÓN } & \multirow[t]{2}{*}{ ACCIONES } & \multicolumn{2}{|c|}{ PERSONAL } & \multicolumn{2}{|c|}{$\begin{array}{l}\text { PERSONAS BENEFICIADAS } \\
\text { (anualmente) }\end{array}$} & \multirow[t]{2}{*}{$\begin{array}{l}\text { INSTANCIAS DE } \\
\text { FINANCIAMIENTO }\end{array}$} & \multirow[t]{2}{*}{$\begin{array}{l}\text { GASTO ANUAL } \\
\text { (\$ mexicanos) }\end{array}$} \\
\hline & & & Total & $\begin{array}{l}\text { Edad } \\
\text { (prom. años) }\end{array}$ & Total & $\begin{array}{c}\text { Adultos mayores } \\
(\%)\end{array}$ & & \\
\hline $\begin{array}{l}\text { Banco de } \\
\text { Alimentos } \\
\text { de Culiacán }\end{array}$ & $\begin{array}{l}\text { Otorgar asistencia } \\
\text { humanitaria y } \\
\text { alimentaria a } \\
\text { personas } \\
\text { marginadas. }\end{array}$ & $\begin{array}{l}\text { Acopio de alimentos } \\
\text { que desechan los } \\
\text { industriales, comercian- } \\
\text { tes y agricultores, para } \\
\text { preparar y distribuir } \\
\text { despensas de } \\
\text { alimentos. }\end{array}$ & 12 & 30 & 18.500 & 26,0 & $\begin{array}{l}\text { Cuota de recuperación. } \\
\text { Gubernamental. } \\
\text { Iniciativa privada. } \\
\text { OSC nacional. } \\
\text { Junta de asistencia } \\
\text { privada estatal. } \\
\text { Ciudadanos. }\end{array}$ & $\$ 9.000 .000$ \\
\hline $\begin{array}{l}\text { Déjalos ir } \\
\text { con Amor }\end{array}$ & $\begin{array}{l}\text { Dar servicios de } \\
\text { calidad a } \\
\text { pacientes graves y } \\
\text { terminales y a sus } \\
\text { familiares. }\end{array}$ & $\begin{array}{l}\text { Apoyo tanatológico, } \\
\text { psicológico y médico. }\end{array}$ & 10 & 35 & 236 & 80,0 & $\begin{array}{l}\text { Iniciativa privada. } \\
\text { Junta de asistencia } \\
\text { privada estatal. } \\
\text { Donador privado. } \\
\text { Prestación de servicios. } \\
\text { Fundación nacional. }\end{array}$ & $\$ 700.000$ \\
\hline $\begin{array}{l}\text { Asociación } \\
\text { de Diabetes } \\
\text { de Sinaloa }\end{array}$ & $\begin{array}{l}\text { Prevenir y educar } \\
\text { en diabetes a } \\
\text { personas sin } \\
\text { acceso a servicios } \\
\text { de salud. }\end{array}$ & $\begin{array}{l}\text { Campañas de } \\
\text { información, donacio- } \\
\text { nes de medicamentos, } \\
\text { publicaciones } \\
\text { científicas periodísticas. }\end{array}$ & 5 & 40 & 600 & 15,0 & $\begin{array}{l}\text { Sorteos. } \\
\text { Aportaciones de } \\
\text { los miembros. } \\
\text { Iniciativa privada. } \\
\text { Cuota de recuperación. }\end{array}$ & $\$ 1.080 .000$ \\
\hline
\end{tabular}

Fuente: Elaboración propia. 
médicos privados se vieron afectados porque si les llegaban cinco pacientes al día ahora les llegan dos y ellos hacen una estrategia en el Colegio [de médicos] para no permitir que ningún médico local trabaje con nosotros."

Esa falta de apoyo está asociada a la percepción que se tiene de las OSC, a las cuales se suele considerar como instancias altruistas cuyo personal es voluntario y no requiere retribución alguna (23). De esta forma se asume que la responsabilidad de atender a ciertos grupos de la población o de enfocarse en determinados temas es de quien tiene un interés personal al respecto. No obstante, para poder atender a grupos como los AM, es necesario un personal de tiempo completo con un salario competitivo y de insumos e infraestructura suficientes.

Con relación al marco institucional, actualmente, la Ley Federal de Fomento a las Actividades Realizadas por Organizaciones de la Sociedad Civil es el principal marco regulatorio de sus actividades y estructura. Su principal objetivo es establecer los derechos y las obligaciones de las OSC que cumplen con los requisitos para ser objeto de fomento a sus actividades a través de apoyos y estímulos que otorga el gobierno federal; sin embargo, todo lo que tiene que ver con obligaciones y beneficios fiscales lo establece la Secretaría de Hacienda y Crédito Público, y las Juntas de Asistencia Privada son las instancias que determinan, a nivel estatal, qué OSC pueden recibir beneficios fiscales de acuerdo a las actividades que realizan. Por lo tanto, no existen lineamientos homogéneos al respecto y no todas las juntas están estructuradas y operan de la misma forma:

"En nuestro país no hay una diferencia entre una asociación no lucrativa y la lucrativa, hay otros países como Chile, en donde tú abres una asociación civil y automáticamente los bancos te dejan que pongas un botecito para donativos, el periódico (la radio y la televisión) te dan un espacio, la compañía de luz te disminuye el costo, teléfono, agua, predial, todos te dan apoyo, aquí aun siendo asociación civil, todos los servicios se pagan como lucrativa."

\section{DISCUSIÓN Y CONCLUSIONES}

La evidente falta de OSC en estas ciudades, dedicadas específicamente a los AM, es un dato importante que consideramos como resultado del estudio en sí mismo, y que Ilama la atención sobre el hecho de que el acelerado envejecimiento poblacional no está vinculado con un número también creciente de OSC dirigidas a cubrir sus necesidades; suponemos que esta situación es un reflejo de lo que sucede a nivel nacional, ya que como se mencionó, las ciudades elegidas reflejan de alguna manera la diversidad del país.

Con base en estudios previos se sabe que en México el apoyo gubernamental a los AM es incipiente; la escasa producción de literatura científica sobre el tema sugiere también una falta de interés sobre este grupo poblacional. Será necesario un viraje en este sentido para preparar y concientizar a los distintos sectores de la sociedad mexicana, para que estén listos ante el enorme reto que representa el incremento exponencial de los AM en los próximos años.

Todas las OSC trabajan en temas relacionados con la calidad de vida de personas empobrecidas: alimentos, útiles escolares, asilo, alfabetización, capacitación técnica y en valores humanos, además de microcréditos. La salud física y mental forma parte importante de las acciones que Ilevan a cabo estas OSC y es el interés central en las tres que trabajan para la atención de algún problema específico como la diabetes y la salud visual; también dan apoyo permanente en salud dos OSC cuya población mayoritaria son los AM (Club de Amigos de la Tercera Edad y Déjalos ir con Amor). La atención tanatológica y los problemas de salud física y visual son comunes entre los AM, en el caso de las enfermedades de los ojos, es frecuente que se presenten asociadas al propio deterioro senil o bien a otras enfermedades metabólicas y/o ocupacionales (27). Esto explica por qué tres de estas seis OSC son las que atienden a un mayor porcentaje de AM.

En general, el concepto de salud que está implícito en estas acciones se reduce a una visión biomédica de atención a la enfermedad, siendo casi nulas las acciones de prevención y promoción. Las implicaciones de esta visión, tratándose de la 
población envejecida, reflejan una representación social de la vejez que predomina en términos de comorbilidad, improductividad y que demanda acciones inmediatas más que a largo plazo, en virtud de la esperanza de vida.

De las cinco OSC restantes únicamente la que en su origen se dedicaba totalmente a los AM (Ancianos y sus Amigos) tiene permanentemente atención en salud. Las dos OSC que buscan un cambio sustentable (tercera generación) no tienen como grupo objetivo a los AM e incluyen acciones en salud de forma esporádica.

Esta diferenciación sugiere que existe una visión paternalista y estigmatizada compartida en el sector, derivada de la percepción de que los viejos son un sector de la población sin potencialidad productiva y que sus principales necesidades son de índole asistencialista como asilo, alimentación y atención a sus enfermedades.

El personal que trabaja en las OSC es mayoritariamente femenino; este mismo hallazgo ha sido explicado por algunos autores como resultado de los valores que sustentan las entidades no lucrativas, que al contrario de la estructura del mercado laboral capitalista tradicional y al sector público estatal, se basan en la solidaridad, igualdad, ayuda mutua, no discriminación, libertad laboral de entrada y salida de los socios, todo lo cual crea un ambiente propicio para las mujeres (28). Desde una perspectiva de género (29), a la identidad femenina se asocian características tales como el servicio y el cuidado hacia otros. Las OSC se distinguen por su labor básicamente altruista, de manera que se constituyen en espacios atractivos para las mujeres, en virtud de la correspondencia que guarda con la identidad antes descrita.

Los resultados de este estudio reflejan la importancia de las OSC a través de programas que mejoran la calidad de vida de las personas empobrecidas, incluidos los AM. Los beneficios de las OSC estudiadas son tanto cuantitativos, por la cantidad de recursos que movilizan y su cobertura, como cualitativos, por su papel en temas como participación, autogestión y empoderamiento de la población. El impacto de las OSC es diferencial y esto corresponde tanto a cuestiones internas como externas. Internamente un problema generalizado es la dificultad para asegurar un financiamiento permanente, sin que esto les reste autonomía sobre los temas y poblaciones a las cuales van a beneficiar. Muchos de los problemas relacionados con este tema se podrán solucionar en la medida en que las OSC aumenten su autosuficiencia económica; en este sentido sería importante documentar y difundir los casos exitosos existentes de autosuficiencia como los logrados por las dos clínicas de salud visual del estudio.

Por otro lado, si bien es cierto que las OSC asistencialistas realizan un papel fundamental al solucionar problemas inmediatos de la población, también lo es el hecho de que muchos de los problemas relacionados con la pobreza del país son de carácter estructural, por lo que sería necesario una mayor cantidad de organizaciones de tercera generación trabajando para lograr la sustentabilidad de los cambios en las comunidades. Para fomentar una mayor proporción de OSC de tercera generación con autosuficiencia económica, es necesario ofrecer una mayor capacitación sobre ambos temas en los múltiples cursos y talleres enfocados a este sector.

También se identificó que las políticas de los partidos, gobiernos estatales e iniciativa privada no favorecen el desarrollo de las OSC. El hecho de que México tenga uno de los sectores más reducidos de Latinoamérica puede deberse a esta situación, así como también a una limitada difusión de sus servicios por falta de capacidad de respuesta y al propio reconocimiento de las OSC de que su trabajo es insuficiente para contener la pobreza.

Como lo demuestran nuestros resultados, al igual que el resto de América Latina, la capacidad de las OSC estudiadas de distribuir bienes públicos es limitada, por lo que el desafío político es cómo aumentar su capacidad para transformarse en socios autónomos del Estado, el cual debe seguir teniendo un papel central en la creación o distribución de la riqueza. De igual manera, una mejor articulación entre los productores de demanda (la sociedad civil) y los generadores de resultados (los partidos y el gobierno) constituye una cuestión estratégica fundamental para fortalecer la democracia (19). Como señalan Bresser y Cunnill, si se acepta que la mejor opción política para la descentralización administrativa es por medio de la SC, las OSC deben ser tratadas como prioridad de gobierno y no como un socio 
eventual de segunda clase, destinado a las tareas complementarias. Esto significa un tratamiento Institucional en donde se dé financiamiento y se regulen las actividades que desarrollan las OSC (3).

Para que el sector continúe su desarroIlo es necesario incrementar y mejorar los estímulos fiscales y recursos públicos gubernamentales, con reglas claras que aseguren la equidad. Se considera necesario y urgente realizar, tanto a nivel estatal como nacional, campañas de fomento a las OSC para concientizar a los ciudadanos, iniciativa privada, partidos políticos, medios de comunicación, académicos y gobiernos de sus beneficios, logrando obtener su apoyo y fomentando relaciones de alianza más que de competitividad entre estos sectores, lo que las acercaría más a la categoría de tercera generación. En el caso de Morelos y Guerrero, un cambio en este sentido sería la institución y funcionamiento adecuado de los organismos autónomos encargados de fomentar las actividades de las OSC, como sucede en muchos otros estados.

\section{AGRADECIMIENTOS}

Este estudio fue financiado por el Instituto Nacional de Desarrollo Social de México: proyecto CS-09-IVI-057-05. Expresamos nuestro agradecimiento a todos los miembros de las OSC que participaron como informantes en el presente estudio.

\section{CONFLICTO DE INTERESES}

No hay conflictos de intereses por parte de los autores del trabajo.

\section{NOTAS FINALES}

a. El Seguro Popular es un programa recientemente implementado con el objetivo de ofrecer atención médica a la población que carece de seguridad social.

\section{REFERENCIAS BIBLIOGRÁFICAS}

1. González-Vázquez TT. Desmitificando la globalización neoliberal y sus críticos altermundistas. En: Salazar R. Chávez A, coordinadores. Sentido y contrasentido en América Latina. Argentina: Elaleph.com; 2008. p. 53-91.

2. Cansino C, Ortiz S. Nuevos enfoques sobre la sociedad civil. Metapolítica. 1997;1(2):211-226.
3. Bresser C, Cunill N. Entre el Estado y el mercado. Lo público no estatal. En: Bresser C, Cunill N, editores. Lo público no estatal en la reforma del estado. Caracas: CLAD, Buenos Aires: Paidós; 1998. p. 25-56.

4. Cunill N. Balance de la participación ciudadana en las políticas sociales. Propuesta de un marco analítico. En: Ziccardi A, coordinadora. Participación ciudadana y políticas sociales en el 
ámbito local. México DF: UNAM, Instituto de Investigaciones sociales, Consejo Mexicano de Ciencias Sociales, Instituto Nacional de Desarrollo Social; 2004. p. 57-75.

5. Cohen J, Arato A. Sociedad civil y teoría política. México DF: Fondo de Cultura Económica; 2002.

6. Torres B. Las organizaciones no gubernamentales: avances de investigación sobre sus características y actuación. En: Méndez JL, coordinador. Organizaciones civiles y políticas públicas en México y Centroamérica. México DF: Porrúa, ISTR; 1998. p. 35-61.

7. Salamon LM, Sokolowski W, List R. Global Civil Society: an overview. En: Salamon LM, Wojciech S, Sokolowski W, editors. Global Civil Society: Dimensions of the Nonprofit Sector. Bloomfield: Kumarian Press; 2004. vol 2, p. 3-60.

8. Girardo C, coordinadora. La profesionalización de las OSC en México: actores y estrategias. Toluca: El Colegio Mexiquense; 2007.

9. Verduzco G, List R, Salamon LM. México. En: Salamon LM, Helmut K, Anheier, List R, Toepler S, Sokolowski W. La sociedad civil global: Las dimensiones del sector no lucrativo. Madrid: Fundación BBVA; 2001. p. 517-534.

10. Charry $\mathrm{Cl}$. Geoestadísticas de las ONGs en México hoy. Polis: Investigación y Análisis Sociopolítico y Psicosocial. 2003;(1):185-204.

11. Kalache A, Lunenfeld B. La salud y el hombre que envejece. Ginebra: Organización Mundial de la Salud; 2001.

12. Consejo Nacional de Población. Encuesta Nacional de la Dinámica Demográfica 2006 [Internet]. México DF: CONAPO: 2006 [citado 25 Mar 2009]. Disponible en: http://www.conapo.gob.mx/encuesta/Enadid/index.html

13. Consejo Nacional de Población. Comunicado de prensa 04/05 [Internet]. México DF: SEGOB; 27 Feb 2005 [citado 9 Dic 2008]. Disponible en: http://www.conapo.gob.mx/prensa/2005/042005.pdf

14. Wong R. Envejecimiento en áreas urbanas marginadas de México: condiciones mixtas de privilegio y desventaja. En: Salgado de Snyder VN, Wong $\mathrm{R}$, editoras. Envejecimiento, pobreza y salud en población urbana: Un estudio en cuatro ciudades de México. Cuernavaca: INSP; 2006. p. 25-35.

15. Guzmán JM. El proceso de envejecimiento demográfico en América Latina y el Caribe. En:
Envejecimiento y Desarrollo en América Latina y el Caribe. Santiago de Chile: Naciones Unidas; 2002. p. 9-12. (CEPAL Serie Población y Desarrollo No. 28)

16. Márquez M., Pelcastre B. y Salgado de Snyder N. Recursos económicos y derechohabiencia en la vejez en contextos de pobreza urbana. En: Salgado de Snyder N, Wong R. Envejecimiento, pobreza y salud en población urbana. Un estudio en cuatro ciudades de México. Cuernavaca: INSP; 2006. p. 71-84.

17. Hubanks A, Kuy W. Quality of life assessment an annotated bibliography. Ginebra: WHO, MNH, PSF; 1994.

18. Vera-Noriega JA, Sotelo TI. Bienestar y redes de apoyo social en población de la tercera edad. La Psicología Social en México. 2004;(10):95-102.

19. Sorj B. ¿Pueden las ONG reemplazar al Estado? Sociedad Civil y Estado en América Latina. Nuevas Sociedad. 2007;(210):126-140.

20. Centro Mexicano para la Filantropía. Búsqueda de instituciones [Internet]. México DF [citado 10 Oct 2007]. Disponible en: http://cemefi.org/directorio/buscasl.php

21. Salgado de Snyder VN, Wong R, editores. Envejeciendo en la pobreza: género, salud y calidad de vida. Cuernavaca: INSP; 2003.

22. Salgado de Snyder VN, Wong R, editoras. Envejecimiento, pobreza y salud en población urbana: Un estudio en cuatro ciudades de México. Cuernavaca: INSP; 2006.

23. Gómez-Jauregui J. The Feasibility of Government Partnerships with NGOs in the Reproductive Health Field in Mexico. Reprod Health Matters. 2004;12(24):42-55.

24. Grindle $M$, Hinderbrand $M$. Building Sustainable capacity in the public sector: What can be done? Public Administration and Development. 1995;(15):441-463.

25. Hall R. Organizations. Structures, Process and Outcomes. New Jersey: Prentice Hall; 1999.

26. Gronbjerg K. The Vital importance of funding relations: sectoral and organizational perspectives. En: Understanding nonprofit Funding. San Francisco: Jossey-Bass; 1994. p. 3-25.

28. Casparis H, Lindsley K, Bressler NB. Surgery for cataracts in people with age-related macular 
degeneration. Cochrane Database of Systematic Reviews. 2009;Jan 21(1):CD006757.

29. Ribas A, Sajardo A. La desigual participación de hombres y mujeres en la economía social: teorías explicativas. Revista de Economía Pública, Social y Cooperativa. 2004;(50):77-103.
30. Lamas $M$, compiladora. El género: la construcción cultural de la diferencia sexual. Ciudad de México: PUEG; 1996.

\section{FORMA DE CITAR}

González Vázquez TT, Márquez Serrano M, Treviño Siller S, Gómez-Jauregui J, Pelcastre-Villafuerte BE. La capacidad de las Organizaciones de la Sociedad Civil para mejorar la calidad de vida de los adultos mayores: un estudio cualitativo en zonas urbanas empobrecidas de México. Salud Colectiva. 2010;6(2):181-194.

Recibido el 1 de diciembre de 2009

Versión final presentada el 15 de abril de 2010

Aprobado el 26 de abril de 2010 